\title{
Correlative TEM and Atom Probe Tomography - A Case Study on Structural Materials for Fusion Reactors
}

\author{
Stephan Krämer ${ }^{1}$, Peter Wells ${ }^{2}$, Christian Oberdorfer ${ }^{3}$ and Robert G. Odette ${ }^{2}$ \\ 1. Center for Nanoscale Systems, Harvard University, Cambridge, USA. \\ 2. Materials Department, University of California, Santa Barbara, USA. \\ 3. Department of Materials Science and Engineering, Ohio State University, Columbus, USA.
}

Both atom probe (AP) and transmission electron microscopy (TEM) provide three-dimensional (3D) chemical and structural information about a material on the nanometer to near-atomic scale. The sample geometry is conducive to record both AP and TEM tomograms on the identical piece, thus giving the opportunity to combine the strength of both techniques, i.e. high chemical sensitivity of AP and TEM's capability to visualize crystallographic and morphologic defects. It is demonstrated in this paper that such an approach can lead both to a better understanding of materials properties as well as help analyse potential artefacts in atom probe reconstruction.

Study objects are two steels that are potential candidates as structural components in fusion reactors. One of the many materials' challenges for realizing energy production via fusion is the necessity to manage high concentrations of helium, which is formed during neutron-alpha reactions. Helium atoms are insoluble in steel and cluster to form nm-sized pressurized bubbles. Atom probe tips were prepared onto Omniprobe grids using focused ion beam milling in a FEI Helios Nanolab 600, transferred to a FEI Tecnai T20 operated at $200 \mathrm{kV}$ using the Hummingbird tomography holder followed by destructive atom probe analysis in a Cameca LEAP 3000.

Correlation of the aforementioned techniques relies on the faithful reconstruction of either 3D distribution, as well as their proper scaling and alignment. Major issues in the case of TEM tomograms are in the given case low signal-to-noise ratio and artefacts due to Bragg diffraction. Noise sensitivity is improved by employing an algebraic reconstruction scheme. The second issue could be overcome via slight under focusing during bright field imaging. The interference contrast at pore boundaries, as shown in figure 1(a), was strong enough to allow analysis of pores with diameters down to two nanometers.

Atom probe tomograms depend, among many other factors, sensitively on the actual shape of the sample and are affected by local inhomogeneities in the field evaporation. The recorded electron tomograms readily provide an accurate measure for the evolution of the tip radius along the needle axis. The second effect is demonstrated in figure 1 which shows TEM and atom probe data of a steel that had formed $\mathrm{He}$ pores with diameters of order 10nm. The atom probe reconstruction in figure 1(c) shows an unphysical increase in Fe counts near locations of pores as illustrated in the overlap of TEM and AP data in 1(d). Furthermore the Fe excess regions are elongated. TAPSIM simulations [1] applied to this geometrical problem show very similar features (figures 1(e) and (f)), an important step towards faithful reconstruction of the actual distribution of elements.

The second steel, a nanoferritic alloy contains a high density of Y-Ti-O nanoclusters that trap helium into a high density of sub-critical nm-scale interface bubbles. Neither technique by itself was able to provide satisfactory information about the large-scale correlation of $\mathrm{He}$ bubble formation and nanocluster location. Figure 2 shows a corresponding TEM-AP data pair. The results show 
unequivocally a one-to-one correlation of $\mathrm{He}$ bubble and nano oxide cluster location. Geometric parameters such as pore density and diameter will further provide essential data to better understand and model helium transport in those material systems and help improve lifetime predictions.

\section{References:}

[1] C Oberdorfer et al, Ultramicroscopy 128 (2013), p. 55.
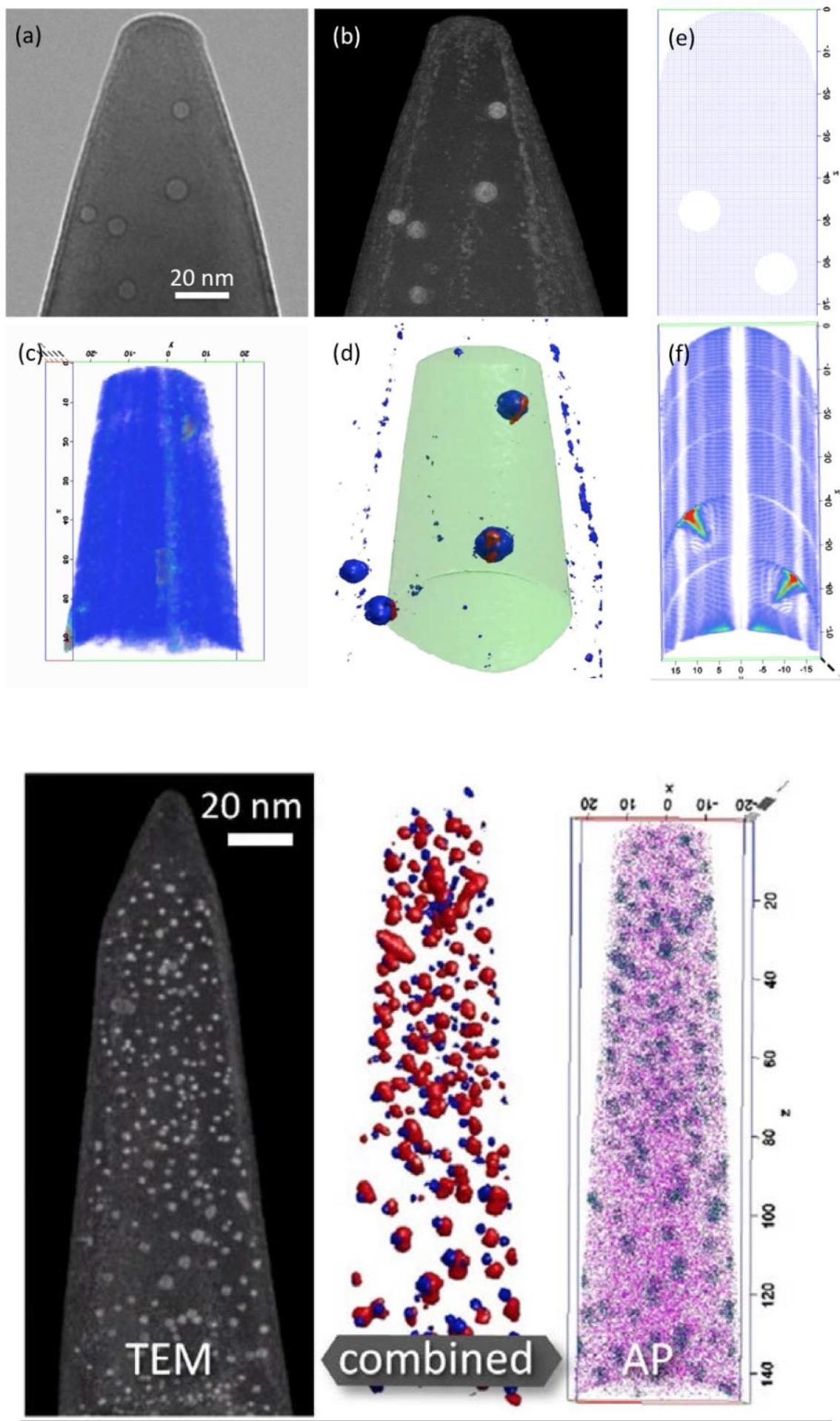

Figure 1. (a) Bright field TEM image of a steel containing pores of diameter $\sim 10 \mathrm{~nm}$, (b) projected semi-transparent view of the corresponding $3 \mathrm{D}$ reconstruction, (c) atom probe (AP) reconstruction, (d) overlap of $3 \mathrm{D}$ atom probe (red) and TEM data (blue), (the extent of the AP is marked green), (e) atomic model for TAPSIM simulation, (f) correspond-ding solution for the iron distribution.
Figure 2. TEM tomogram (left) showing cavity locations in white, a 2D slice of AP tomogram (right) showing location of $\mathrm{TiO}$ ions in gray and locations of $\mathrm{Fe}$ atoms in pink, and correlation of the two datasets (center) via surface rendering of bubbles (blue) and oxides (red). 